(n)

trobertivier Journal of Nonlinear Mathematical Physics

\title{
Semi-discrete hyperbolic equations admitting five dimensional characteristic $x$-ring
}

Kostyantyn Zheltukhin, Natalya Zheltukhina

To cite this article: Kostyantyn Zheltukhin, Natalya Zheltukhina (2016) Semi-discrete hyperbolic equations admitting five dimensional characteristic $x$-ring, Journal of Nonlinear Mathematical Physics 23:3, 351-367, DOI:

https://doi.org/10.1080/14029251.2016.1199497

To link to this article: https://doi.org/10.1080/14029251.2016.1199497

Published online: 04 January 2021 


\title{
Semi-discrete hyperbolic equations admitting five dimensional characteristic $x$-ring
}

\author{
Kostyantyn Zheltukhin \\ Department of Mathematics, Faculty of Science, \\ Middle East Technical University 06531 Ankara, Turkey \\ zheltukh@metu.edu.tr \\ Natalya Zheltukhina \\ Department of Mathematics, Faculty of Science, \\ Bilkent University, 06800 Ankara, Turkey \\ natalya@fen.bilkent.edu.tr
}

Received 3 March 2016

Accepted 18 April 2016

\begin{abstract}
The necessary and sufficient conditions for a hyperbolic semi-discrete equation to have five dimensional characteristic $x$-ring are derived. For any given chain, the derived conditions are easily verifiable by straightforward calculations.
\end{abstract}

Keywords: Hyperbolic semi-discrete equations; Darboux integrability; Characteristic ring.

2000 Mathematics Subject Classification: 37K10, 17B80, 39A99

\section{Introduction}

In the present paper we are considering integrability of hyperbolic type semi-discrete equations. There exist many different approaches to define and classify integrable equations: symmetry approach, Peinlevé analysis, method of algebraic entropy and other methods. For classification of hyperbolic type equations the approach based on the notions of characteristic rings turns out to be very effective.

The notion of a characteristic ring was introduced by Shabat to classify hyperbolic systems of exponential type

$$
u_{x y}^{i}=e^{\left(a_{i 1} u_{1}+a_{i 2} u_{2}+\cdots+a_{i n} u_{n}\right)} \quad i=1,2, \ldots n,
$$

such system has a finite dimensional characteristic ring if and only if $A=\left(a_{i, j}\right)$ is a Cartan matrix of a semi-simple Lie algebra, see [1]. Then in [2] it was shown that a system of hyperbolic equations

$$
u_{x y}^{i}=f^{i}\left(u_{1}, u_{2}, \ldots u_{n}\right) \quad i=1,2, \ldots n
$$

can be integrated in quadratures if its characteristic ring is finite dimensional.

Zhiber and his collaborators considered application of the characteristic ring to classification problems of general hyperbolic equations

$$
u_{x y}=f\left(u, u_{x}, u_{y}\right) .
$$


In particular the classification of equations $\mathrm{Eq}(1.3)$ admitting two dimensional, three dimensional or four dimensional (for some special form of function $f$ ) characteristic rings was considered in [3][5]. For other classification results based on the notion of the characteristic ring see [6]- [9] and a review paper [10].

Later Habibullin extended the notion of characteristic ring to semi-discrete and discrete equations and applied this notion to solve different classification problems for such equations (see [11][19]).

Let us give necessary definitions. Consider a hyperbolic type semi-discrete equation

$$
t_{1 x}=f\left(x, t, t_{1}, t_{x}\right)
$$

where the function $t(n, x)$ depends on discrete variable $n$ and continuous variable $x$. We use the following notations $t_{x}=\frac{\partial}{\partial x} t, t_{1}=t(n+1, x)$, and $t_{[k]}=\frac{\partial^{k}}{\partial x^{k}} t$, where $k \in \mathbb{N}$ and $t_{m}=t(n+m, x)$, $m \in \mathbb{Z}$.

Definition 1.1. A function $F\left(x, t, t_{1}, \ldots, t_{k}\right)$ is called an $x$-integral of the equation Eq.(1.4) if

$$
D_{x} F\left(x, t, t_{1}, \ldots, t_{k}\right)=0
$$

for all solutions of Eq.(1.4). The operator $D_{x}$ is the total derivative with respect to $x$. A function $G\left(x, t, t_{x}, \ldots, t_{[m]}\right)$ is called an $n$-integral of the equation Eq.(1.4) if

$$
D G\left(x, t, t_{x}, \ldots, t_{[m]}\right)=G\left(x, t, t_{x}, \ldots, t_{[m]}\right)
$$

for all solutions of Eq.(1.4).

The equation Eq.(1.4) is called Darboux integrable if it admits non trivial $x$ - and $n$-integrals (see [12]).

Example 1.1. For example the equation

$$
t_{1 x}=\frac{t t_{1}}{t_{x}}
$$

has an $x$-integral $F=\frac{t_{2}}{t}$ and an $n$-integral $I=\frac{t}{t_{x}}+\frac{t_{x}}{t}$. Hence the equation is Darboux integrable.

We note that a Darboux integrable equation can be reduced to a pair of ordinary equations: ordinary differential equation and ordinary difference equation.

In [12] an effective criterion for the existence of $x$ - and $n$-integrals was given.

Theorem 1.1. [12] An equation Eq.(1.4) admits a non-trivial $x$-integral if and only if its characteristic $x$-ring is of finite dimension.

An equation Eq.(1.4) admits a non-trivial n-integral if and only if its characteristic n-ring is of finite dimension.

It is generally believed that a finite dimensional characteristic $x$-ring can not have dimension larger than five. The examples of Darboux integrable semi-discrete equations known to us support this hypothesis. On the other hand one can construct examples of Darboux integrable semi-discrete equations with characteristic $n$-ring of an arbitrary large finite dimension. So we study semi-discrete equation Eq.(1.4) with five dimensional characteristic $x$-ring. The case of three and four dimensional rings were considered in [15] and [21] respectively. 
In general it is not easy to determined the dimension of the characteristic ring. In our paper we give the necessary and sufficient conditions for the characteristic $x$-ring to be five dimensional. The derived conditions are checked by straightforward calculations and can be effectively used to determine if the the characteristic $x$-ring is five dimensional. We also present two examples of equations that have five dimensional characteristic $x$-ring.

The paper is organized as follows. In Section 2 we introduce the characteristic $x$-ring for a general equation Eq.(1.4). In Section 3 we derive necessary and sufficient conditions for the characteristic $x$-ring to be five dimensional and give two example of an equation with five dimensional $x$-ring. Equation Eq.(3.23) was introduced in [20]. The second equation Eq.(3.25) we believe to be new. Note that equation Eq.(1.5) possesses four dimensional characteristic $x$-ring.

\section{Characteristic ring of a hyperbolic type equation}

The characteristic $x$-ring $L_{x}$ of the equation Eq.(1.4) is generated by two vector fields (see [12])

$$
X=\frac{\partial}{\partial t_{x}}
$$

and

$$
K=\frac{\partial}{\partial x}+t_{x} \frac{\partial}{\partial t}+f \frac{\partial}{\partial t_{1}}+g \frac{\partial}{\partial t_{-1}}+f_{1} \frac{\partial}{\partial t_{2}}+\ldots
$$

where function $g$ is determined by

$$
t_{-1 x}=g\left(x, t_{-1}, t, t_{x}\right) .
$$

To obtain above equality we apply $D^{-1}$ to Eq.(1.4) and then solve the resulting equation for $t_{-1 x}$.

Let us introduce some vector fields from $L_{x}$.

$$
C_{1}=[X, K] \quad \text { and } \quad C_{n}=\left[X, C_{n-1}\right] \quad n=2,3, \ldots
$$

and

$$
Z_{1}=\left[K, C_{1}\right] \quad \text { and } \quad Z_{n}=\left[K, Z_{n-1}\right] \quad n=2,3, \ldots
$$

To write this vector fields it is convenient to define the following quantities

$$
p=\frac{f_{x}+t_{x} f_{t}+f f_{t_{1}}}{f_{t_{x}}}, \quad v=f_{t}+f_{t_{x}} f_{t_{1}}, \quad w=f_{x t_{x}}+t_{x} f_{t_{x} t}+f f_{t_{x} t_{1}}, \quad h=f_{t_{x} t_{x} t_{x}} f_{t_{x}}-3 f_{t_{x} t_{x}}^{2} .
$$

We have

$$
\begin{aligned}
& C_{1}=\frac{\partial}{\partial t}+f_{t_{x}} \frac{\partial}{\partial t_{1}}+g_{t_{x}} \frac{\partial}{\partial t_{-1}}+\ldots \\
& C_{2}=f_{t_{x} t_{x}} \frac{\partial}{\partial t_{1}}+g_{t_{x} t_{x}} \frac{\partial}{\partial t_{-1}}+\ldots \\
& Z_{1}=(w-v) \frac{\partial}{\partial t_{1}}+\ldots
\end{aligned}
$$

and so on.

Let us determine what vectors can form a basis of $L_{x}$ assuming that $\operatorname{dim} L_{x}=5$. 
First assume that $f_{t_{x} t_{x}} \neq 0$. We have that vector fields $X, K, C_{1}$ and $C_{2}$ are linearly independent. Also, as was shown in [21], if $C_{3}$ and $Z_{1}$ belong to the linear span of $X, K, C_{1}$ and $C_{2}$ then $L_{x}$ is four dimensional algebra. To have a five dimensional algebra one of the vectors $C_{3}, Z_{1}$ must be linearly independent of $X, K, C_{1}$ and $C_{2}$. Hence if $f_{t_{x} t_{x}} \neq 0$ the five dimensional algebra $L_{x}$ is generated either by $X, K, C_{1}, C_{2}$ and $Z_{1}$ or by $X, K, C_{1}, C_{2}$ and $C_{3}$.

If $f_{t_{x} t_{x}}=0$ then $C_{n}=0, n=2,3 \ldots$ and the algebra is spanned by $X, K, C_{1}, Z_{1}$ and $Z_{2}$.

To check that a vector admits the expansion with respect to a particular basis we use the following Remark.

Remark 2.1. One can check equalities between vector fields using the automorphism $D() D^{-1}$. Direct calculations show that

$$
D X D^{-1}=\frac{1}{f_{t_{x}}} X, \quad D K D^{-1}=K-p X .
$$

The images of other vector fields under this automorphism can be obtained by commuting $D X D^{-1}$ and $D K D^{-1}$.

\section{Five dimensional characteristic $x$-rings}

\subsection{Case 1}

Let us find conditions for the characteristic algebra $L_{x}$ to be generated by linearly independent vector fields $X, K, C_{1}, C_{2}$ and $Z_{1}$. (We assume that $f_{t_{x} t_{x}} \neq 0$.)

As the next lemma shows to check that vector fields $X, K, C_{1}, C_{2}$ and $Z_{1}$ form a basis of $L_{x}$ it is enough to check that the vectors fields $C_{3},\left[K, C_{2}\right]$ and $\left[K, Z_{1}\right]$ have unique expansions. Also we note that if $C_{3},\left[K, C_{2}\right]$ and $\left[K, Z_{1}\right]$ can be expended with respect to $X, K, C_{1}, C_{2}$ and $Z_{1}$ then they are linear combinations of $C_{2}$ and $Z_{1}$ only

$$
\begin{gathered}
C_{3}=\alpha C_{2}+\beta Z_{1}, \\
{\left[K, C_{2}\right]=\gamma C_{2}+\mu Z_{1},} \\
{\left[K, Z_{1}\right]=\eta C_{2}+\sigma Z_{1},}
\end{gathered}
$$

for some functions $\alpha, \beta, \gamma, \mu, \eta$ and $\sigma$. This follows from the fact that

$$
X=\frac{\partial}{\partial t_{x}}, \quad K=\frac{\partial}{\partial x}+t_{x} \frac{\partial}{\partial t}+\ldots \quad C_{1}=\frac{\partial}{\partial t}+\ldots
$$

but vector fields $C_{3},\left[K, C_{2}\right]$ and $\left[K, Z_{1}\right]$ do not contain $\frac{\partial}{\partial t_{x}}, \frac{\partial}{\partial x}$ and $\frac{\partial}{\partial t}$ in their representations.

Lemma 3.1. The vector fields $X, K, C_{1}, C_{2}$ and $Z_{1}$ form a basis of the characteristic $x$-ring $L_{x}$ if and only if vectors fields $C_{3},\left[K, C_{2}\right]$ and $\left[K, Z_{1}\right]$ admit a unique linear representations with respect to the basis vector fields.

Proof. We need to prove that if vectors fields $C_{3},\left[K, C_{2}\right]$ and $\left[K, Z_{1}\right]$ admit a unique linear representations with respect to the basis then all other commutators of the basis vector fields, in particular $\left[Z_{1}, X\right],\left[C_{1}, C_{2}\right],\left[C_{1}, Z_{1}\right]$ and $\left[C_{2}, Z_{1}\right]$, also admit unique linear representations. Assume that $C_{3}$, 
$\left[K, C_{2}\right]$ and $\left[K, Z_{1}\right]$ have unique linear representation with respect to the basis vector fields. That is equalities $\mathrm{Eq}(3.1)-\mathrm{Eq}(3.3)$ hold.

Let us show that vector field $\left[Z_{1}, X\right]$, has unique expansion with respect to the basis. Using definitions of vector fields $Z_{1}, C_{1}, C_{2}$ and Jacobi identity we can write

$$
\left[Z_{1}, X\right]=\left[\left[K, C_{1}\right], X\right]=-\left(\left[\left[C_{1}, X\right], K\right]+\left[[X, K], C_{1}\right]\right)=\left[C_{2}, K\right]-\left[C_{1}, C_{1}\right]=-\left[K, C_{2}\right] .
$$

Thus from $\mathrm{Eq}(3.2)$ it follows that

$$
\left[Z_{1}, X\right]=-\left[K, C_{2}\right]=-\gamma C_{2}-\mu Z_{1} .
$$

Let us show that the vector field $\left[C_{1}, C_{2}\right]$ has unique expansion with respect to the basis.

Using the definition of vector fields $C_{1}, C_{3}$ and Jacobi identity we can write

$$
\left[C_{1}, C_{2}\right]=\left[[X, K], C_{2}\right]=-\left(\left[\left[K, C_{2}\right], X\right]+\left[\left[C_{2}, X\right], K\right]\right)=\left[X,\left[C_{2}, K\right]\right]+\left[C_{3}, K\right]
$$

Then from $\mathrm{Eq}(3.1)$ and $\mathrm{Eq}(3.3)$ it follows that

$$
\begin{aligned}
& {\left[C_{1}, C_{2}\right]=\left[X, \gamma C_{2}+\mu Z_{1}\right]+\left[\alpha C_{2}+\beta Z_{1}, K\right]} \\
& =X(\gamma) C_{2}+\gamma C_{3}+X(\mu) Z_{1}+\mu\left[X, Z_{1}\right] \\
& \quad-K(\alpha) C_{2}+\alpha\left[C_{2}, K\right]-K(\beta) Z_{1}+\beta\left[Z_{1}, K\right] \\
& =X(\gamma) C_{2}+\gamma\left(\alpha C_{2}+\beta Z_{1}\right)+X(\mu) Z_{1}+\mu\left(\gamma C_{2}+\mu Z_{1}\right) \\
& \quad-K(\alpha) C_{2}-\alpha\left(\gamma C_{2}+\mu Z_{1}\right)-K(\beta) Z_{1}-\beta\left(\eta C_{2}+\sigma Z_{1}\right) .
\end{aligned}
$$

Hence,

$$
\left[C_{1}, C_{2}\right]=e C_{2}+q Z_{1},
$$

where $e=(X(\gamma)+\gamma \alpha+\mu \gamma-K(\alpha)-\alpha \gamma-\beta \eta)$ and $q=\left(\gamma \beta+X(\mu)+\mu^{2}-\alpha \mu-K(\beta)-\beta \sigma\right)$.

Let us show that the vector field $\left[C_{1}, Z_{1}\right]$ has unique expansion with respect to the basis. Using the definition of $C_{1}$ and Jacobi identity we can write

$$
\left[C_{1}, Z_{1}\right]=\left[[X, K], Z_{1}\right]=-\left(\left[\left[K, Z_{1}\right], X\right]+\left[\left[Z_{1}, X\right], K\right]\right)
$$

Using equalities $\mathrm{Eq}(3.3)$ and $\mathrm{Eq}(3.4)$ we have

$$
\begin{aligned}
& {\left[C_{1}, Z_{1}\right]=\left[X, \eta C_{2}+\sigma Z_{1}\right]+\left[\gamma C_{2}+\mu Z_{1}, K\right]} \\
& =X(\eta) C_{2}+\eta C_{3}+X(\sigma) Z_{1}+\sigma\left[X, Z_{1}\right] \\
& \quad-K(\gamma) C_{2}+\gamma\left[C_{2}, K\right]-K(\mu) Z_{1}+\mu\left[Z_{1}, K\right] \\
& =X(\eta) C_{2}+\eta\left(\alpha C_{2}+\beta Z_{1}\right)+X(\sigma) Z_{1}+\sigma\left(\gamma C_{2}+\mu Z_{1}\right) \\
& \quad-K(\gamma) C_{2}-\gamma\left(\gamma C_{2}+\mu Z_{1}\right)-K(\mu) Z_{1}-\mu\left(\eta C_{2}+\sigma Z_{1}\right)
\end{aligned}
$$

Hence,

$$
\left[C_{1}, Z_{1}\right]=r C_{2}+s Z_{1},
$$

where $r=\left(X(\eta)+\alpha \eta+\gamma \sigma-K(\gamma)-\gamma^{2}-\mu \eta\right)$ and $s=(\alpha \beta+X(\sigma)+\mu \sigma-\gamma \mu-K(\mu)-\mu \sigma)$. 
Let us show that the vector field $\left[C_{2}, Z_{1}\right]$ has unique expansion with respect to the basis. Using the definition of $C_{2}$ and Jacobi identity we can write

$$
\left[C_{2}, Z_{1}\right]=\left[\left[X, C_{1}\right], Z_{1}\right]=-\left(\left[\left[C_{1}, Z_{1}\right], X\right]+\left[\left[Z_{1}, X\right], C_{1}\right]\right)
$$

Using definition of $C_{3}$ and equalities $\mathrm{Eq}(3.1)$ and $\mathrm{Eq}(3.4)-\mathrm{Eq}(3.6)$ we have

$$
\begin{aligned}
& {\left[C_{2}, Z_{1}\right]=\left[X, r C_{2}+s Z_{1}\right]+\left[\gamma C_{2}+\mu Z_{1}, C_{1}\right]} \\
& \begin{array}{r}
=X(r) C_{2}+r C_{3}+X(s) Z_{1}+s\left[X, Z_{1}\right] \\
\quad-C_{1}(\gamma) C_{2}+\gamma\left[C_{2}, C_{1}\right]-C_{1}(\mu) Z_{1}+\mu\left[Z_{1}, C_{1}\right] \\
=X(r) C_{2}+r\left(\alpha C_{2}+\beta Z_{1}\right)+X(s) Z_{1}+s\left(\gamma C_{2}+\mu Z_{1}\right) \\
\quad-C_{1}(\gamma) C_{2}-\gamma\left(e C_{2}+q Z_{1}\right)-C_{1}(\mu) Z_{1}-\mu\left(r C_{2}+s Z_{1}\right)
\end{array}
\end{aligned}
$$

Hence,

$$
\left[C_{2}, Z_{1}\right]=m C_{2}+n Z_{1}
$$

where $m=\left(X(r)+\alpha r+s \gamma-C_{1}(\gamma)-\gamma e-\mu r\right)$ and $n=\left(r \beta+X(s)+s \mu-\gamma q-C_{1}(\mu)-\mu s\right)$.

Now let us find under what conditions the equalities $\mathrm{Eq}(3.1)-\mathrm{Eq}(3.3)$ hold.

Remark 3.1. Each of the equalities Eq.(3.1), Eq.(3.2) and Eq.(3.3) leads to a certain system for the coefficients and one obtains the coefficients by solving the corresponding system. Hence the vector fields $X, K, C_{1}, C_{2}$ and $Z_{1}$ form a basis if and only if the solutions of the systems, that determine coefficients, exist and unique.

This remark holds for other cases as well.

Let us write the systems corresponding to equalities Eq.(3.1), Eq.(3.2) and Eq.(3.3).

Lemma 3.2. The equality Eq.(3.1) holds if and only if the coefficients $\alpha$ and $\beta$ satisfy the following system

$$
\begin{aligned}
& E_{11}^{(1)} \beta+E_{12}^{(1)}(D \beta) \quad=F_{1}^{(1)} \\
& E_{22}^{(1)}(D \beta)+E_{23}^{(1)} \alpha+E_{24}^{(1)}(D \alpha)=F_{2}^{(1)} \\
& E_{32}^{(1)}(D \beta)+\quad E_{34}^{(1)}(D \alpha)=F_{3}^{(1)}
\end{aligned}
$$

where

$$
\begin{gathered}
E_{11}^{(1)}=\frac{1}{f_{t_{x}}^{2}}, \quad E_{12}^{(1)}=-1, \quad F_{1}^{1}=0, \quad E_{22}^{(1)}=p, \quad E_{23}^{(1)}=\frac{1}{f_{t_{x}}^{2}}, \quad E_{24}^{(1)}=-\frac{1}{f_{t_{x}}}, \quad F_{2}^{(1)}=\frac{3 f_{t_{x} t_{x}}}{f_{t_{x}}^{3}}, \\
E_{32}^{(1)}=w-v-p f_{t_{x} t_{x}}, \quad E_{34}^{(1)}=\frac{f_{t_{x} t_{x}}}{f_{t_{x}}}, \quad F_{3}^{(1)}=\frac{h}{f_{t_{x}}^{3}} .
\end{gathered}
$$


Proof. Applying the automorphism $D(\cdot) D^{-1}$ to Eq.(3.1) we get

$$
D C_{3} D^{-1}=(D \alpha) D C_{2} D^{-1}+(D \beta) D Z_{1} D^{-1} .
$$

Direct calculations show that

$$
\begin{gathered}
D C_{2} D^{-1}=\frac{1}{f_{t_{x}}^{2}} C_{2}-\frac{f_{t_{x} t_{x}}}{f_{t_{x}}^{3}} C_{1}+\frac{f_{t_{x} t_{x}} f_{t}}{f_{t_{x}}^{4}} X, \\
D C_{3} D^{-1}=\frac{1}{f_{t_{x}}^{3}} C_{3}-\frac{3 f_{t_{x} t_{x}}}{f_{t_{x}}^{4}} C_{2}-\frac{h}{f_{t_{x}}^{5}}\left(C_{1}-\frac{f_{t}}{f_{t_{x}}} X\right), \\
D Z_{1} D^{-1}=\frac{1}{f_{t_{x}}} Z_{1}-\frac{p}{f_{t_{x}}} C_{2}+\left(\frac{v-w+p f_{t_{x} t_{x}}}{f_{t_{x}}^{2}}\right)\left(C_{1}-\frac{f_{t}}{f_{t_{x}}} X\right) .
\end{gathered}
$$

Substituting these expressions for $D C_{3} D^{-1}, D C_{2} D^{-1}, D Z_{1} D^{-1}$ into Eq.(3.9) and comparing coefficients of $C_{1}, C_{2}$ and $Z_{1}$ we obtain Eq.(3.8).

Lemma 3.3. The equality Eq.(3.2) holds if and only if the coefficients $\gamma$ and $\mu$ satisfy the following system

$$
\begin{aligned}
& E_{11}^{(2)} \mu+E_{12}^{(2)}(D \mu) \quad=F_{1}^{(2)} \\
& E_{22}^{(2)}(D \mu)+E_{23}^{(2)} \gamma+E_{24}^{(2)}(D \gamma)=F_{2}^{(2)} \\
& E_{32}^{(2)}(D \mu)+\quad E_{34}^{(2)}(D \gamma)=F_{3}^{(2)}
\end{aligned}
$$

where

$$
\begin{gathered}
E_{11}^{(2)}=\frac{1}{f_{t_{x}}}, \quad E_{12}^{(2)}=-1, \quad F_{1}^{(2)}=\frac{f_{t_{x} t_{x}}}{f_{t_{x}}^{2}}+\frac{p \beta}{f_{t_{x}}}, \quad E_{22}^{(2)}=p, \quad E_{23}^{(2)}=\frac{1}{f_{t_{x}}}, \\
E_{24}^{(2)}=-\frac{1}{f_{t_{x}}}, \quad F_{2}^{(2)}=\frac{2 w-p\left(3 f_{t_{x} t_{x}}-f_{t_{x}} \alpha\right)}{f_{t_{x}}^{2}}, \quad E_{32}^{(2)}=w-v-f_{t_{x} t_{x}} p \quad E_{34}^{(2)}=\frac{f_{t_{x} t_{x}}}{f_{t_{x}}}, \\
F_{3}^{(2)}=\frac{-3 f_{t_{x} t_{x}} w+f_{t} f_{t_{x} t_{x}}-p h}{f_{t_{x}}^{2}}+\frac{f_{x t_{x} t_{x}}+t_{x} f_{t t_{x} t_{x}}+f f_{t_{x} t_{x} t_{1}}}{f_{t_{x}}}
\end{gathered}
$$

Proof. Applying the automorphism $D(\cdot) D^{-1}$ to Eq.(3.2) we get

$$
D\left[K, C_{2}\right] D^{-1}=(D \gamma) D C_{2} D^{-1}+(D \mu) D Z_{1} D^{-1}
$$

Direct calculations show that

$$
\begin{aligned}
& D\left[K, C_{2}\right] D^{-1}=\frac{1}{f_{t_{x}}^{2}}\left[K, C_{2}\right]+\frac{3 p f_{t_{x} t_{x}}-2 w}{f_{t_{x}}^{3}} C_{2}-\frac{p}{f_{t_{x}}^{2}} C_{3}-\frac{f_{t_{x} t_{x}}}{f_{t_{x}}^{3}} Z_{1} \\
& +\left(\frac{3 f_{t_{x} t_{x}} w-f_{t_{x} t_{x}} f_{t}+p h}{f_{t_{x}}^{4}}-\frac{f_{x t_{x} t_{x}}+t_{x} f_{t t_{x} t_{x}}+f f_{t_{1} t_{x} t_{x}}}{f_{t_{x}}^{3}}\right) C_{1}+\ldots X
\end{aligned}
$$

Substituting the expressions for $D\left[K, C_{2}\right] D^{-1}, D C_{2} D^{-1}, D Z_{1} D^{-1}$ into Eq.(3.11) and comparing coefficients of $C_{1}, C_{2}$ and $Z_{1}$ we obtain Eq.(3.10). 
Lemma 3.4. The equality Eq.(3.3) holds if and only if the coefficients $\eta$ and $\sigma$ satisfy the following system

$$
\begin{aligned}
& E_{11}^{(3)} \sigma+E_{12}^{(3)}(D \sigma) \quad=F_{1}^{(3)} \\
& E_{22}^{(3)}(D \sigma)+E_{23}^{(3)} \eta+E_{24}^{(3)}(D \eta)=F_{2}^{(3)} \\
& E_{32}^{(3)}(D \sigma)+\quad E_{34}^{(3)}(D \eta)=F_{3}^{(3)}
\end{aligned}
$$

where

$$
\begin{gathered}
E_{11}^{(3)}=1, \quad E_{12}^{(3)}=-1, \quad F_{1}^{(3)}=p\left(2 \mu-p \beta-\frac{2 f_{t_{x} t_{x}}}{f_{t_{x}}}\right)+\frac{2 w-v}{f_{t_{x}}}, \\
E_{22}^{(3)}=p, \quad E_{23}^{(3)}=1, \quad E_{24}^{(3)}=-\frac{1}{f_{t_{x}}}, \\
F_{2}^{(3)}=p(2 \gamma-p \alpha)+K(p)+\frac{3 p^{2} f_{t_{x} t_{x}}-3 p w}{f_{t_{x}}}, \quad E_{32}^{(3)}=-f_{t_{x} t_{x}} p-v+w, \quad E_{34}^{(3)}=\frac{f_{t_{x} t_{x}}}{f_{t_{x}}}, \\
F_{3}^{(3)}=-(K-p X)\left\{f_{t_{x} t_{x}} p+v-w\right\}+\left(f_{t_{x} t_{x}} p+v-w\right) \frac{2 w-f_{t}-2 p f_{t_{x} t_{x}}}{f_{t_{x}}}
\end{gathered}
$$

Proof. Applying the automorphism $D(\cdot) D^{-1}$ to Eq.(3.3) we get

$$
D\left[K, Z_{1}\right] D^{-1}=(D \eta) D C_{2} D^{-1}+(D \sigma) D Z_{1} D^{-1}
$$

Direct calculations show that

$$
\begin{gathered}
D\left[K, Z_{1}\right] D^{-1}=\left(\frac{\sigma-2 p \mu+p^{2} \beta}{f_{t_{x}}}+\frac{2 p f_{t_{x} t_{x}}+v-2 w}{f_{t_{x}}^{2}}\right) Z_{1} \\
+\left(-(K-p X)\left\{\frac{p}{f_{t_{x}}}\right\}+\frac{\eta-2 p \gamma+p^{2} \alpha}{f_{t_{x}}}-\frac{p\left(p f_{t_{x} t_{x}}+v-w\right)}{f_{t_{x}}^{2}}\right) C_{2} \\
+\left((K-p X)\left\{\frac{p f_{t_{x_{x}} t_{x}}+v-w}{f_{t_{x}}^{2}}\right\}+\frac{f_{t}\left(p f_{t_{x} t_{x}}+v-w\right)}{f_{t_{x}}^{3}}\right) C_{1}+\ldots X
\end{gathered}
$$

Substituting the expressions for $D\left[K, Z_{1}\right] D^{-1}, D C_{2} D^{-1}, D Z_{1} D^{-1}$ and comparing coefficients of $C_{1}$, $C_{2}$ and $Z_{1}$ we obtain Eq.(3.12).

All the systems in the above lemmas have similar form, in particular,

$$
\begin{aligned}
& E_{11} u+E_{12}(D u) \quad=F_{1} \\
& E_{22}(D u)+E_{23} v+E_{24}(D v)=F_{2} \\
& E_{32}(D u)+\quad E_{34}(D v)=F_{3}
\end{aligned}
$$

where $u, v$ are unknowns.

We need conditions for existence of a unique solution for such systems. The conditions are given in the following lemma. 
Lemma 3.5. The system Eq.(3.13) has a unique solution if $E_{11}, E_{12}, E_{22}, E_{23}, E_{24}, E_{32}, E_{34}$ and $F_{1}$, $F_{2}, F_{3}$ satisfy

$$
\left(-E_{11} E_{22} E_{34}\left(D^{-1} E_{34}\right)+E_{11} E_{24} E_{32}\left(D^{-1} E_{34}\right)-E_{12} E_{23} E_{34}\left(D^{-1} E_{32}\right)\right) \neq 0
$$

and

$$
(D H)=\frac{F_{1}}{E_{12}}-\frac{E_{11}}{E_{12}} H
$$

where

$$
\begin{array}{r}
H=\left(\left(F_{1} E_{24} E_{32}-F_{1} E_{22} E_{34}-F_{2} E_{12} E_{34}-F_{3} E_{12} E_{24}\right)\left(D^{-1} E_{34}\right)+\left(D^{-1} F_{3}\right) E_{12} E_{23} E_{34}\right) \\
\left(-E_{11} E_{22} E_{34}\left(D^{-1} E_{34}\right)+E_{11} E_{24} E_{32}\left(D^{-1} E_{34}\right)-E_{12} E_{23} E_{34}\left(D^{-1} E_{32}\right)\right)^{-1}
\end{array}
$$

Proof. In the system Eq.(3.13) the coefficients and variables depend on the discrete variable $n \in \mathbb{Z}$. So we can rewrite the system as follows

$$
\begin{aligned}
& E_{11}(n) u(n)+E_{12}(n) u(n+1) \quad=F_{1}(n) \\
& E_{22}(n) u(n+1)+E_{23}(n) v(n)+E_{24}(n) v(n+1)=F_{2}(n) \\
& E_{32}(n) u(n+1)+\quad E_{34}(n) v(n+1)=F_{3}(n)
\end{aligned}
$$

The above equalities must hold for all values of $n$. Applying $D^{-1}$ to the last equation above we obtain

$$
E_{32}(n-1) u(n)+E_{34}(n-1) v(n)=F_{3}(n-1) .
$$

Now we have a linear system to find $u(n), v(n), u(n+1)$ and $v(n+1)$ independently. The system has a unique solution if condition Eq.(3.14) holds. Solving the system we find

$$
u(n)=H, u(n+1)=\frac{F_{1}}{E_{12}}-\frac{E_{11}}{E_{12}} H
$$

and

$$
v(n)=\frac{\left(D^{-1} F_{3}\right)}{\left(D^{-1} E_{34}\right)}-\frac{\left(D^{-1} E_{32}\right)}{\left(D^{-1} E_{34}\right)} H, v(n+1)=\frac{F_{3}}{E_{34}}-\frac{E_{32} F_{1}}{E_{34} E_{12}}+\frac{E_{32} E_{11}}{E_{34} E_{12}} H
$$

The condition Eq.(3.14) shows that $D u(n)=u(n+1)$ and $D v(n)=v(n+1)$. Hence the system Eq.(3.17) has a unique solution.

Now we can give necessary and sufficient conditions for the algebra to be generated by vector fields $X, K, C_{1}, C_{2}$ and $Z_{1}$.

Theorem 3.1. The characteristic $x$-ring of Eq.(1.4) is generated by vector fields $X, K, C_{1}, C_{2}$ and $Z_{1}$ if and only if the following conditions are satisfied 


$$
\left(-E_{11}^{(i)} E_{22}^{(i)} E_{34}^{(i)}\left(D^{-1} E_{34}^{(i)}\right)+E_{11}^{(i)} E_{24}^{(i)} E_{32}^{(i)}\left(D^{-1} E_{34}^{(i)}\right)-E_{12}^{(i)} E_{23}^{(i)} E_{34}^{(i)}\left(D^{-1} E_{32}^{(i)}\right)\right) \neq 0
$$

and

$$
\left(D H^{(i)}\right)=\frac{F_{1}^{(i)}}{E_{12}^{(i)}}-\frac{E_{11}^{(i)}}{E_{12}^{(i)}} H^{(i)},
$$

where

$$
\begin{gathered}
H^{(i)}=\left(\left(F_{1}^{(i)} E_{24}^{(i)} E_{32}^{(i)}-F_{1}^{(i)} E_{22}^{(i)} E_{34}^{(i)}-F_{2}^{(i)} E_{12}^{(i)} E_{34}^{(i)}-F_{3}^{(i)} E_{12}^{(i)} E_{24}^{(i)}\right)\left(D^{-1} E_{34}^{(i)}\right)+\left(D^{-1} F_{3}^{(i)}\right) E_{12}^{(i)} E_{23}^{(i)} E_{34}^{(i)}\right) \\
\left(-E_{11}^{(i)} E_{22}^{(i)} E_{34}^{(i)}\left(D^{-1} E_{34}^{(i)}\right)+E_{11}^{(i)} E_{24}^{(i)} E_{32}^{(i)}\left(D^{-1} E_{34}^{(i)}\right)-E_{12}^{(i)} E_{23}^{(i)} E_{34}^{(i)}\left(D^{-1} E_{32}^{(i)}\right)\right)^{-1}
\end{gathered}
$$

where $i=1,2,3$.

Proof. By Lemma 3.5 the conditions Eq.(3.20), Eq.(3.21) imply that the systems Eq.(3.8), Eq.(3.10) and Eq.(3.12) have unique solutions. Hence equalities Eq.(3.1), Eq.(3.2) and Eq.(3.3) hold and the characteristic ring $L_{x}$ is generated by vector fields $X, K, C_{1}, C_{2}$ and $Z_{1}$.

Example 3.1. Consider an equation

$$
t_{1 x} t_{x}=t+t_{1}
$$

introduced by Adler and Startsev in [20]. For this equation one can easily check that the conditions of the Theorem 3.1 are satisfied. Hence the characteristic ring $L_{x}$ is five dimensional and generated by vector fields $X, K, C_{1}, C_{2}$ and $Z_{1}$. We have

$$
C_{3}=-\frac{3}{t_{x}} C_{2}, \quad\left[K, C_{2}\right]=-\frac{1}{t_{x}} Z_{1}, \quad\left[K, Z_{1}\right]=-\frac{1}{t_{x}} Z_{1} .
$$

The $x$-integral and $n$-integral for the above equation are

$$
F=\frac{\left(u_{3}-u_{1}\right)\left(u_{2}-u\right)}{\left(u_{2}+u_{1}\right)}, \quad I=\frac{\left(u_{x x}-1\right)^{2}}{u_{x}^{2}} .
$$

Example 3.2. Consider an equation

$$
t_{1 x}=\cosh \left(t_{1}-t\right) t_{x}+\sinh \left(t_{1}-t\right) \sqrt{t_{x}^{2}-1}
$$

For this equation one can easily check that the conditions of the Theorem 3.1 are satisfied. Hence the characteristic ring $L_{x}$ is five dimensional and generated by vector fields $X, K, C_{1}, C_{2}$ and $Z_{1}$. We have

$$
C_{3}=-\frac{3 t_{x}}{t_{x}^{2}-1} C_{2}, \quad\left[K, C_{2}\right]=-\frac{t_{x}}{t_{x}^{2}-1} Z_{1}, \quad\left[K, Z_{1}\right]=\left(t_{x}^{2}-1\right)^{\frac{1}{2}} Z_{1}
$$

The $x$-integral and $n$-integral for the above equation are

$$
\hat{F}=\frac{\left(e^{t_{2}}-e^{t_{1}}\right)\left(e^{t_{3}}-e^{t}\right)}{\left(e^{t_{2}}-e^{t}\right)\left(e^{t_{3}}-e^{t_{1}}\right)}, \quad \hat{I}=e^{-t}\left(t_{x}+\sqrt{t_{x}^{2}-1}\right) .
$$




\subsection{Case 2}

Let us find conditions for the characteristic algebra $L_{x}$ to be generated by vector fields $X, K, C_{1}, C_{2}$ and $C_{3}$. (We assume that $f_{t_{x} t_{x}} \neq 0$.)

As the next lemma shows to check that vector fields $X, K, C_{1}, C_{2}$ and $C_{3}$ form a basis of $L_{x}$ it is enough to check that the vectors fields $Z_{1},\left[C_{1}, C_{2}\right]$ and $C_{4}$ have unique expansions. Also we note that if $Z_{1},\left[C_{1}, C_{2}\right]$ and $C_{4}$ can be expended with respect to $X, K, C_{1}, C_{2}$ and $C_{3}$ then

$$
\begin{gathered}
Z_{1}=\tilde{\lambda} C_{2}, \\
{\left[C_{1}, C_{2}\right]=\tilde{\alpha} C_{2}+\tilde{\beta} C_{3},} \\
C_{4}=\tilde{\mu} C_{2}+\tilde{\eta} C_{3} .
\end{gathered}
$$

for some functions $\tilde{\lambda}, \tilde{\alpha}, \tilde{\beta}, \tilde{\mu}$ and $\tilde{\eta}$. This follows from the form of $Z_{1},\left[C_{1}, C_{2}\right]$ and $C_{4}$. Note that if $Z_{1}=\tilde{\lambda}_{1} C_{2}+\tilde{\lambda}_{2} C_{3}$ with $\tilde{\lambda}_{2} \neq 0$ we have the Case 1 .

Lemma 3.6. The vector fields $X, K, C_{1}, C_{2}$ and $C_{3}$ form a basis of the characteristic $x$-ring $L_{x}$ if and only if vectors fields $Z_{1},\left[C_{1}, C_{2}\right]$ and $C_{4}$ admit unique linear representations with respect to the basis vector fields.

The above Lemma is proved in the same way as Lemma 3.1.

Let us write the systems corresponding to equalities Eq.(3.28) and (3.29). The condition for the equality Eq.(3.27) was obtained in [21].

Lemma 3.7. The equality Eq.(3.28) holds if and only if the coefficients $\tilde{\alpha}$ and $\tilde{\beta}$ satisfy the following system

$$
\begin{aligned}
& \tilde{E}_{11}^{(2)} \tilde{\beta}+\tilde{E}_{12}^{(2)}(D \tilde{\beta}) \quad=\tilde{F}_{1}^{(2)} \\
& \tilde{E}_{22}^{(2)}(D \tilde{\beta})+\tilde{E}_{23}^{(2)} \tilde{\alpha}+E_{24}^{(2)}(D \tilde{\alpha})=\tilde{F}_{2}^{(2)} \\
& \tilde{E}_{32}^{(2)}(D \tilde{\beta})+\quad \tilde{E}_{34}^{(2)}(D \tilde{\alpha})=\tilde{F}_{3}^{(2)}
\end{aligned}
$$

where

$$
\begin{gathered}
\tilde{E}_{11}^{(2)}=1, \quad \tilde{E}_{12}^{(2)}=-1, \quad \tilde{F}_{1}^{(2)}=\frac{v}{f_{t_{x}}}, \\
\tilde{E}_{22}^{(2)}=\frac{3 f_{t_{x} t_{x}}}{f_{t_{x}}^{2}}, \quad \tilde{E}_{23}^{(2)}=\frac{1}{f_{t_{x}}}, \quad \tilde{E}_{24}^{(2)}=-1, \quad \tilde{F}_{2}^{(2)}=\frac{2\left(f_{t t_{x}}+f_{t_{x}} f_{t_{1} t_{x}}\right)}{f_{t_{x}}^{2}}-\frac{3 f_{t_{x} t_{x}} v-f_{t_{x} t_{x}} f_{t}}{f_{t_{x}}^{3}} \\
\tilde{E}_{32}^{(2)}=\frac{h}{f_{t_{x}}^{2}}, \quad \tilde{E}_{34}^{(2)}=f_{t_{x} t_{x}}, \quad \tilde{F}_{3}^{(2)}=\frac{f_{t t_{x} t_{x}}+f_{t_{x}} f_{t_{1} t_{x} t_{x}}-3 f_{t_{x} t_{x}} f_{t_{1} t_{x}}}{f_{t_{x}}}-\frac{2 f_{t_{x} t_{x}} f_{t t_{x}}}{f_{t_{x}}^{2}}-\frac{f_{x_{x} t_{x}}^{2} f_{t}+v h}{f_{t_{x}}^{3}} . \\
\text { Co-published by Atlantis Press and Taylor \& Francis } \\
\text { Copyright: the authors }
\end{gathered}
$$


Proof. Applying the automorphism $D(\cdot) D^{-1}$ to Eq.(3.28) we get

$$
D\left[C_{1}, C_{2}\right] D^{-1}=(D \tilde{\alpha}) D C_{2} D^{-1}+(D \tilde{\beta}) D C_{3} D^{-1} .
$$

Direct calculations show that

$$
\begin{gathered}
D\left[C_{1}, C_{2}\right] D^{-1}=\frac{1}{f_{t_{x}}^{3}}\left[C_{1}, C_{2}\right]-\frac{v}{f_{t_{x}}^{4}} C_{3}+\left(-\frac{2\left(f_{t t_{x}}+f_{t_{x}} f_{t_{1} t_{x}}\right)}{f_{t_{x}}^{4}}-\frac{f_{t_{x} t_{x}} f_{t}}{f_{t_{x}}^{5}}+\frac{3 v f_{t_{x} t_{x}}}{f_{t_{x}}^{5}}\right) C_{2} \\
+\left(\frac{f_{t_{x} t_{x}} f_{t_{1} t_{x}}}{f_{t_{x}}^{4}}-\frac{1}{f_{t_{x}}} C_{1}\left(\frac{f_{t_{x} t_{x}}}{f_{t_{x}}^{3}}\right)-\frac{f_{t_{x} t_{x}}\left(f_{t t_{x}}+f_{t_{x}} f_{t_{1} t_{x}}\right)}{f_{t_{x}}^{5}}+\frac{f_{t_{x} t_{x}}^{2} f_{t}}{f_{t_{x}}^{6}}+\frac{v h}{f_{t_{x}}^{6}}\right) C_{1}+\ldots X
\end{gathered}
$$

Substituting the expressions for $D\left[C_{1}, C_{2}\right] D^{-1}, D C_{2} D^{-1}, D C_{3} D^{-1}$ and comparing coefficients of $C_{1}$, $C_{2}$ and $C_{3}$ we obtain Eq.(3.30).

Lemma 3.8. The equality Eq.(3.29) holds if and only if the coefficients $\tilde{\mu}$ and $\tilde{\eta}$ satisfy the following system

$$
\begin{aligned}
\tilde{E}_{11}^{(3)} \tilde{\eta}+\tilde{E}_{12}^{(3)}(D \tilde{\eta}) & =\tilde{F}_{1}^{(3)} \\
\tilde{E}_{22}^{(3)}(D \tilde{\eta})+\tilde{E}_{23}^{(3)} \tilde{\mu}+\tilde{E}_{24}^{(3)}(D \tilde{\mu}) & =\tilde{F}_{2}^{(3)} \\
\tilde{E}_{32}^{(3)}(D \tilde{\eta})+\tilde{E}_{34}^{(3)}(D \tilde{\mu}) & =\tilde{F}_{3}^{(3)}
\end{aligned}
$$

where

$$
\begin{gathered}
\tilde{E}_{11}^{(3)}=\frac{1}{f_{t_{x}}}, \quad \tilde{E}_{12}^{(3)}=-1, \quad \tilde{F}_{1}^{(3)}=\frac{6 f_{t_{x} t_{x}}}{f_{t_{x}}^{2}}, \\
\tilde{E}_{22}^{(3)}=\frac{3 f_{t_{x} t_{x}}}{f_{t_{x}}^{2}}, \quad \tilde{E}_{23}^{(3)}=\frac{1}{f_{t_{x}}^{2}}, \quad \tilde{E}_{24}^{(3)}=-1, \quad \tilde{F}_{2}^{(3)}=\frac{4 h-3 f_{t_{x} t_{x}}^{2}}{f_{t_{x}}^{4}}, \\
\tilde{E}_{32}^{(3)}=\frac{h}{f_{t_{x}}^{2}}, \quad \tilde{E}_{34}^{(3)}=f_{t_{x} t_{x}}, \quad \tilde{F}_{3}^{(2)}=\frac{f_{t_{x} t_{x} t_{x} t_{x}} f_{t_{x}}-5 f_{t_{x} t_{x}} f_{t_{x} t_{x} t_{x}}}{f_{t_{x}}^{3}}-\frac{5 f_{t_{x} t_{x}} h}{f_{t_{x}}^{4}} .
\end{gathered}
$$

Proof. Applying the automorphism $D(\cdot) D^{-1}$ to Eq.(3.29) we get

$$
D C_{4} D^{-1}=(D \tilde{\mu}) D C_{2} D^{-1}+(D \tilde{\eta}) D C_{3} D^{-1} .
$$

Direct calculations show that

$$
D C_{4} D^{-1}=\frac{1}{f_{t_{x}}^{4}} C_{4}-\frac{6 f_{t_{x} t_{x}}}{f_{t_{x}}^{5}} C_{3}-\left(\frac{3\left(f_{t_{x} t_{x} t_{x}} f_{t_{x}}-4 f_{t_{x} t_{x}}^{2}\right)}{f_{t_{x}}^{6}}+\frac{h}{f_{t_{x}}^{6}}\right) C_{2}-\frac{1}{f_{t_{x}}} X\left(\frac{h}{f_{t_{x}}^{5}}\right) C_{1}+\ldots X .
$$

Substituting the expressions for $D C_{4} D^{-1}, D C_{2} D^{-1}, D C_{3} D^{-1}$ and comparing coefficients of $C_{1}, C_{2}$ and $C_{3}$ we obtain Eq.(3.31). 
Theorem 3.2. The characteristic $x$-ring of Eq.1.4 is generated by vector fields $X, K, C_{1}, C_{2}$ and $C_{3}$ if and only if the following conditions are satisfied

$$
\begin{gathered}
D\left(\frac{f_{t_{x} t_{x} t_{x}}}{f_{t_{x} t_{x}}}\right)=\frac{f_{t_{x} t_{x} t_{x}} f_{t_{x}}-3 f_{t_{x} t_{x}}^{2}}{f_{t_{x} t_{x}} f_{t_{x}}^{2}}, \\
\left(-\tilde{E}_{11}^{(i)} \tilde{E}_{22}^{(i)} \tilde{E}_{34}^{(i)}\left(D^{-1} \tilde{E}_{34}^{(i)}\right)+\tilde{E}_{11}^{(i)} \tilde{E}_{24}^{(i)} \tilde{E}_{32}^{(i)}\left(D^{-1} \tilde{E}_{34}^{(i)}\right)-E_{12}^{(i)} E_{23}^{(i)} \tilde{E}_{34}^{(i)}\left(D^{-1} \tilde{E}_{32}^{(i)}\right)\right) \neq 0
\end{gathered}
$$

and

$$
\left(D \tilde{H}^{(i)}\right)=\frac{\tilde{F}_{1}^{(i)}}{\tilde{E}_{12}^{(i)}}-\frac{\tilde{E}_{11}^{(i)}}{\tilde{E}_{12}^{(i)}} \tilde{H}^{(i)},
$$

where

$$
\begin{gathered}
\tilde{H}^{(i)}=\left(\left(\tilde{F}_{1}^{(i)} \tilde{E}_{24}^{(i)} \tilde{E}_{32}^{(i)}-\tilde{F}_{1}^{(i)} \tilde{E}_{22}^{(i)} \tilde{E}_{34}^{(i)}-\tilde{F}_{2}^{(i)} \tilde{E}_{12}^{(i)} \tilde{E}_{34}^{(i)}-\tilde{F}_{3}^{(i)} \tilde{E}_{12}^{(i)} \tilde{E}_{24}^{(i)}\right)\left(D^{-1} \tilde{E}_{34}^{(i)}\right)+\left(D^{-1} \tilde{F}_{3}^{(i)}\right) \tilde{E}_{12}^{(i)} \tilde{E}_{23}^{(i)} \tilde{E}_{34}^{(i)}\right) \\
\left(-\tilde{E}_{11}^{(i)} \tilde{E}_{22}^{(i)} \tilde{E}_{34}^{(i)}\left(D^{-1} \tilde{E}_{34}^{(i)}\right)+\tilde{E}_{11}^{(i)} \tilde{E}_{24}^{(i)} \tilde{E}_{32}^{(i)}\left(D^{-1} \tilde{E}_{34}^{(i)}\right)-E_{12}^{(i)} \tilde{E}_{23}^{(i)} \tilde{E}_{34}^{(i)}\left(D^{-1} \tilde{E}_{32}^{(i)}\right)\right)^{-1}
\end{gathered}
$$

for $i=2,3$.

Proof. The condition Eq.3.32 implies that the equality Eq.(3.27) holds, see [21]. By Lemma 3.5 the conditions Eq.(3.33) and Eq.(3.34) imply that the systems Eq.(3.30) and Eq.(3.31) have unique solutions. Hence equalities Eq.(3.27), Eq.(3.28) and Eq.(3.29) hold and the characteristic ring $L_{x}$ is generated by vector fields $X, K, C_{1}, C_{1}$ and $C_{3}$.

\subsection{Case 3}

Let us find conditions for the characteristic algebra $L_{x}$ to be generated by vector fields $X, K, C_{1}, Z_{1}$ and $Z_{2}$. (We assume that $f_{t_{x} t_{x}}=0$.)

As in the previous cases to check that $X, K, C_{1}, Z_{1}$ and $Z_{2}$ form a basis it is enough to check that $\left[C_{1}, Z_{1}\right]$ and $\left[K, Z_{2}\right]$ have unique expansion. Also we note that if $\left[C_{1}, Z_{1}\right]$ and $\left[K, Z_{2}\right]$ can be expended with respect to $X, K, C_{1}, Z_{1}$ and $Z_{2}$ then

$$
\begin{gathered}
{\left[C_{1}, Z_{1}\right]=\bar{\alpha} Z_{1},} \\
{\left[K, Z_{2}\right]=\bar{\lambda} Z_{1}+\bar{\mu} Z_{2}}
\end{gathered}
$$

for some functions $\bar{\alpha}, \bar{\lambda}$ and $\bar{\mu}$. This follows from the form of $\left[C_{1}, Z_{1}\right]$ and $\left[K, Z_{2}\right]$. In general one should write $\left[C_{1}, Z_{1}\right]=\bar{\alpha} Z_{1}+\bar{\beta} Z_{2}$ but we show that $\bar{\beta}$ is zero in the next lemma.

Lemma 3.9. Let $f_{t_{x} t_{x}}=0$ then if the vector field $\left[C_{1}, Z_{1}\right]$ admits linear representation with respect to vector fields $X, K, C_{1}, Z_{1}$ and $Z_{2}$ then equality $E q(3.36)$ holds. 
Proof. From the form of $\left[C_{1}, Z_{1}\right]$ it follows that $\left[C_{1}, Z_{1}\right]=\bar{\alpha} Z_{1}+\bar{\beta} Z_{2}$. Let us show that $\bar{\beta}$ is zero. We have $f_{t_{x} t_{x}}=0$ and $f_{t_{x} t_{x}}=0$ if and only if

$$
C_{2}=0
$$

Using definition of $Z_{1}, Z_{2}$ and Jacobi identity we have

$$
\left[X, Z_{1}\right]=\left[X,\left[K, C_{1}\right]\right]=-\left[K,\left[C_{1}, X\right]\right]-\left[C_{1},[X, K]\right]=\left[K, C_{2}\right]-\left[C_{1}, C_{1}\right]=0
$$

and

$$
\left[X, Z_{2}\right]=\left[X,\left[K, Z_{1}\right]\right]=-\left[K,\left[Z_{1}, X\right]\right]-\left[Z_{1},[X, K]\right]=\left[C_{1}, Z_{1}\right]
$$

Since $f_{t_{x} t_{x}}=0$ then $f_{t_{x}}$ does not depend on $t_{x}$ and coefficients of vector field

$$
C_{1}=\frac{\partial}{\partial t}+f_{t_{x}} \frac{\partial}{\partial t_{1}}+g_{t_{x}} \frac{\partial}{\partial t_{-1}}+\ldots
$$

do not depend on $t_{x}$. The equality $\left[X, Z_{1}\right]=0$ implies that the coefficients of $Z_{1}$ also do not depend on $t_{x}$. Thus if $\left[C_{1}, Z_{1}\right]=\bar{\alpha} Z_{1}+\bar{\beta} Z_{2}$ then functions $\bar{\alpha}$ and $\bar{\beta}$ do not depend on $t_{x}$, that is $X(\bar{\alpha})=0$ and $X(\bar{\beta})=0$. Consider $\left[X,\left[C_{1}, Z_{1}\right]\right]$, from one hand, by $\operatorname{Eq}(3.38)$ and Eq.(3.39)

$$
\left[X,\left[C_{1}, Z_{1}\right]\right]=-\left[C_{1},\left[Z_{1}, X\right]\right]-\left[Z_{1},\left[X, C_{1}\right]\right]=-\left[C_{1},\left[Z_{1}, X\right]\right]-\left[Z_{1}, C_{2}\right]=0,
$$

from the other hand,

$$
\left[X,\left[C_{1}, Z_{1}\right]\right]=\left[X, \bar{\alpha} Z_{1}+\bar{\beta} Z_{2}\right]=(X(\bar{\alpha})+\bar{\alpha} \bar{\beta}) Z_{1}+\left(X(\bar{\beta})+\bar{\beta}^{2}\right) Z_{2}=\bar{\alpha} \bar{\beta} Z_{1}+\bar{\beta}^{2} Z_{2}
$$

Therefore, $\bar{\alpha} \bar{\beta} Z_{1}+\bar{\beta}^{2} Z_{2}=0$ or $\bar{\beta}=0$.

The next lemma shows that equalities Eq.(3.36) and Eq.(3.37) imply that vector fields $X, K, C_{1}$, $Z_{1}$ and $Z_{2}$ form a basis of $L_{x}$.

Lemma 3.10. The vector fields $X, K, C_{1}, Z_{1}$ and $Z_{2}$ form a basis of the characteristic $x$-ring $L_{x}$ if and only if vectors fields $\left[C_{1}, Z_{1}\right]$ and $\left[K, Z_{2}\right]$ admit a unique linear representations with respect to the basis vector fields.

The above Lemma is proved in the same way as Lemma 3.1.

Let us write the systems corresponding to equalities Eq.(3.36) and Eq.(3.37).

Lemma 3.11. The equality Eq.(3.36) holds if and only if the $\bar{\alpha}$ and (D $\bar{\alpha})$ satisfy the following system

$$
\begin{gathered}
\frac{1}{f_{t_{x}}} \bar{\alpha}-(D \bar{\alpha})=\frac{f_{t t_{x}}+f_{t_{x}} f_{t_{x} t_{1}}}{f_{t_{x}}^{2}}, \\
(v-w)(D \bar{\alpha})=\frac{f_{t t_{x}}+2 f_{t_{x}} f_{t_{x} t_{1}}}{f_{t_{x}}^{2}}(w-v)+\frac{1}{f_{t_{x}}} C_{1}(v-w) .
\end{gathered}
$$


Proof. Applying the automorphism $D(\cdot) D^{-1}$ to Eq.(3.36) we get

$$
D\left[C_{1}, Z_{1}\right] D^{-1}=(D \bar{\alpha}) D Z_{1} D^{-1}
$$

Direct calculations show that if $f_{t_{x} t_{x}}=0$ then

$$
D\left[C_{1}, Z_{1}\right] D^{-1}=\frac{1}{f_{t_{x}}^{2}}\left[C_{1}, Z_{1}\right]-\frac{f_{t t_{x}}+f_{t_{x}} f_{t_{x} t_{1}}}{f_{t_{x}}^{3}} Z_{1}+\frac{1}{f_{t_{x}}^{3}}\left(\frac{f_{t t_{x}}+2 f_{t_{x}} f_{t_{x} t_{1}}}{f_{t_{x}}}(w-v)+C_{1}(v-w)\right) C_{1}+\ldots X
$$

Substituting the expressions for $D\left[C_{1}, Z_{1}\right] D^{-1}, D Z_{1} D^{-1}$ and comparing coefficients before $C_{1}$ and $Z_{1}$ we obtain Eq.(3.41) and Eq.(3.42) .

Lemma 3.12. The equality Eq.(3.37) holds if and only if the coefficients $\bar{\mu}$ and $\bar{\lambda}$ satisfy the following system

$$
\begin{aligned}
& \bar{E}_{11} \bar{\mu}+\bar{E}_{12}(D \bar{\mu}) \quad=\bar{F}_{1} \\
& \bar{E}_{22}(D \bar{\mu})+\bar{E}_{23} \bar{\lambda}+\bar{E}_{24}(D \bar{\lambda})=\bar{F}_{2} \\
& \bar{E}_{32}(D \bar{\mu})+\quad \bar{E}_{34}(D \bar{\lambda})=\bar{F}_{3}
\end{aligned}
$$

where

$$
\begin{gathered}
\bar{E}_{11}=1, \quad \bar{E}_{12}=-1, \quad \bar{F}_{1}=\frac{3 w-v}{f_{t_{x}}}, \\
\bar{E}_{22}=\frac{2 w-v}{f_{t_{x}}}, \quad \bar{E}_{23}=1, \quad \bar{E}_{24}=-1, \\
\bar{F}_{2}=p \bar{\alpha}-f_{t_{x}} K\left(\frac{v-2 w}{f_{t_{x}}^{2}}\right)-\frac{K(v-w)}{f_{t_{x}}}-\frac{2 w(w-v)}{f_{t_{x}}^{2}}-\frac{f_{t}(v-w)}{f_{t_{x}}^{2}}-\frac{p}{f_{t_{x}}}\left(f_{t t_{x}}+f_{t_{x}} f_{t_{x} t_{1}}\right), \\
\bar{F}_{32}=\frac{K(v-w)}{f_{t_{x}}^{2}}-\frac{2 w(v-w)}{f_{t_{x}}^{3}}+\frac{f_{t}(v-w)}{f_{t_{x}}^{3}}, \quad \bar{E}_{34}=\frac{v-w}{f_{t_{x}}^{2}} \\
\left(\frac{K(v-w)}{f_{t_{x}}^{2}}+\frac{2 w(w-v)}{f_{t_{x}}^{3}}+\frac{2 f_{t}(v-w)}{f_{t_{x}}^{3}}\right)+p X\left(\frac{K(w-v)}{f_{t_{x}}^{2}}+\frac{2 w(v-w)}{f_{t_{x}}^{3}}\right) \\
+\frac{2 p f_{t t_{x}}(w-v)-f_{t_{x}}^{2} Z_{1}(p)+f_{t_{x}}(w-v) C_{1}(p)+f_{t}(v-w) X(p)}{f_{t_{x}}^{3}}
\end{gathered}
$$


Proof. Applying the automorphism $D(\cdot) D^{-1}$ to Eq.(3.37) we get

$$
D\left[K, Z_{2}\right] D^{-1}=(D \bar{\lambda}) D Z_{1} D^{-1}+(D \bar{\mu}) D Z_{2} D^{-1} .
$$

Direct calculations show that if $f_{t_{x} t_{x}}=0$ then

$$
D Z_{2} D^{-1}=\frac{1}{f_{t_{x}}} Z_{2}+\frac{v-2 w}{f_{t_{x}}^{2}} Z_{1}+\frac{1}{f_{t_{x}}^{3}}\left(f_{t_{x}} K(v-w)-2 w(v-w)+f_{t}(v-w)\right) C_{1}+\ldots X
$$

and

$$
D\left[K, Z_{2}\right] D^{-1}=\frac{1}{f_{t_{x}}}\left[K, Z_{2}\right]+\frac{v-3 w}{f_{t_{x}}^{2}} Z_{2}+T Z_{1}-\frac{p}{f_{t_{x}}}\left[X, Z_{2}\right]+R C_{1}+\ldots X
$$

where

$$
\begin{gathered}
T=K\left(\frac{v-2 w}{f_{t_{x}}^{2}}\right)+\frac{1}{f_{t_{x}}^{3}}\left(f_{t_{x}} K(v-w)-2 w(v-w)+f_{t}(v-w)+p f_{t_{x}}\left(f_{t_{t_{x}}}+f_{t_{x}} f_{t_{x} t_{1}}\right)\right), \\
R=(K-p X)\left\{\frac{1}{f_{t_{x}}^{3}}\left(f_{t_{x}} K(v-w)-2 w(v-w)+2 f_{t}(v-w)\right)\right\} \\
-\frac{1}{f_{t_{x}}} Z_{1}(p)+\frac{w-v}{f_{t_{x}}^{2}} C_{1}(p)+\frac{f_{t}}{f_{t_{x}}^{3}}(v-w) X(p) .
\end{gathered}
$$

Note that $\left[X, Z_{2}\right]=\left[C_{1}, Z_{1}\right]$. Substituting the expressions for $D\left[K, Z_{2}\right] D^{-1}, D Z_{1} D^{-1}, D Z_{2} D^{-1}$ and comparing coefficients of $C_{1}, Z_{1}$ and $Z_{2}$ we obtain Eq.(3.43).

Theorem 3.3. The characteristic $x$-ring of Eq.1.4 is generated by vector fields $X, K, C_{1}, Z_{1}$ and $Z_{2}$ if and only if the following conditions are satisfied

$$
\begin{gathered}
D\left(-f_{t_{x} t_{x}}+\frac{C_{1}(v-w)}{v-w}\right)=-\frac{f_{t_{x} t}+2 f_{t_{x}} f_{t_{x} t_{1}}}{f_{t_{x}}^{2}}+\frac{C_{1}(v-w)}{f_{t_{x}}(v-w)} \\
\left(-\bar{E}_{11} \bar{E}_{22} \bar{E}_{34}\left(D^{-1} \bar{E}_{34}\right)+\bar{E}_{11} \bar{E}_{24} \bar{E}_{32}\left(D^{-1} \bar{E}_{34}\right)-\bar{E}_{12} \bar{E}_{23} \bar{E}_{34}\left(D^{-1} \bar{E}_{32}\right)\right) \neq 0
\end{gathered}
$$

and

$$
(D \bar{H})=\frac{\bar{F}_{1}}{\bar{E}_{12}}-\frac{\bar{E}_{11}}{\bar{E}_{12}} \bar{H}
$$

where

$$
\begin{array}{r}
\bar{H}=\left(\left(\bar{F}_{1} \bar{E}_{24} \bar{E}_{32}-\bar{F}_{1} \bar{E}_{22} \bar{E}_{34}-\bar{F}_{2} \bar{E}_{12} \bar{E}_{34}-\bar{F}_{3} \bar{E}_{12} \bar{E}_{24}\right)\left(D^{-1} \bar{E}_{34}\right)+\left(D^{-1} \bar{F}_{3}\right) \bar{E}_{12} \bar{E}_{23} \bar{E}_{34}\right) \\
\left(-\bar{E}_{11} \bar{E}_{22} \bar{E}_{34}\left(D^{-1} \bar{E}_{34}\right)+\bar{E}_{11} \bar{E}_{24} \bar{E}_{32}\left(D^{-1} \bar{E}_{34}\right)-\bar{E}_{12} \bar{E}_{23} \bar{E}_{34}\left(D^{-1} \bar{E}_{32}\right)\right)^{-1}
\end{array}
$$

Proof. In Lemma 3.11 we can easily find $\bar{\alpha}$ and $(D \bar{\alpha})$ independently. The condition that $(D \bar{\alpha})$ is the shift of $\bar{\alpha}$ leads to Eq.(3.45). By Lemma 3.5 the conditions Eq.(3.46) and Eq.(3.47) imply that the system Eq.(3.43) have unique solution. Hence equalities Eq.(3.36) and Eq.(3.37) hold and the characteristic ring $L_{x}$ is generated by vector fields $X, K, C_{1}, Z_{1}$ and $Z_{2}$. 


\section{References}

[1] Shabat A. B. and Yamilov R. I. 1981 Exponential systems of type I and Cartan matrices (Russian) Preprint BBAS USSR Ufa

[2] Leznov A.N., Smirnov V.G. and Shabat A.B. Internal symmetry group and integrability conditions for two-dimensional dynamical systems (Russian) Teoret. Mat. Fiz. 51 (1982) 1021.

[3] Zhiber A.V. and Murtazina R.D. On nonlinear hyperbolic equations with characteristic algebra of slow growth (Russian)Vestnik UGATU 7 (2004) 131-136.

[4] Zhiber A.V. and Murtazina R.D. On the characteristic Lie algebras for the equations $u_{x y}=f\left(u, u_{x}\right) J$. Math. Sci. (N. Y.) 151 (2008) 31123122.

[5] Murtazina R. D. Nonlinear hyperbolic equations with characteristic ring of dimension 3 Ufa Math. J. textbf3 (2011) 113118.

[6] Zhiber A.V. and Mukminov F. Kh. Quadratic systems, symmetries, characteristic and complete algebras (Russian) Problems of Mathematical Physics and Asymptotics or their Solutions Ufa, Institute of Mathematics, RAN, (1991) 13-33.

[7] Kostrigina O.S. and Zhiber A.V. Darboux-integrable two-component nonlinear hyperbolic systems of equations. J. Math. Phys. 52 (2011) 033503.

[8] Sokolov V.V. and Zhiber A.V. On the Darboux integrable hyperbolic equations Phys. Lett. A 208 (1995) 303-308.

[9] Zhiber A.V., Sokolov V.V. and Startsev S.Ya. On nonlinear Darboux-integrable hyperbolic equations (Russian) Dokl. Akad. Nauk 343 (1995) 746-748. Zhiber A. V.and Sokolov V. V. Exactly integrable hyperbolic equations of Liouville type Russian Math. Surveys 56 (2001) 61101.

[10] Zhiber A.B., Murtazina R.D., Habibullin I.T. and Shabat A.B. Characteristic Lie rings and integrable models in mathematical physics Ufa Math. J. 4 (2012) 17-85.

[11] Habibullin I.T. Characteristic algebras of fully discrete hyperbolic type equations SIGMA Symmetry Integrability Geom.: Methods Appl. 1 (2005).

[12] Habibullin I.T. and Pekcan A. Characteristic Lie algebra and the classification of semi-discrete models Theoret. and Math. Phys. 151 (2007) 781-790.

[13] Habibullin I.T. Characteristic algebras of discrete equations Difference equations, special functions and orthogonal polynomials Hackensack NJ World Sci. Publ. (2007) 249-257.

[14] Habibullin I.T. and Gudkova E.V. Classification of integrable discrete Klein-Gordon models Physica Scripta bf 81 (2011) 045003.

[15] Habibullin I.T., Zheltukhina N. and Pekcan A. On some algebraic properties of semi-discrete hyperbolic type equations Turkish J. Math. 32 (2008) 277-292.

[16] Habibullin I.T., Zheltukhina N. and Pekcan A. On the classification of Darboux integrable chains $J$. Math. Phys. 49 (2008) 102702.

[17] Habibullin I.T., Zheltukhina N. and Pekcan A. Complete list of Darboux integrable chains of the form $t_{1 x}=t_{x}+d(t, t 1)$ J. Math. Phys. 50 (2009) 102710.

[18] Habibullin I.T., Zheltukhina N. and Sakieva A. On Darboux-integrable semi-discrete chains J. Phys. A 43 (2010) 434017.

[19] Habibullin I.T. and Zheltukhina N. Discretization of Liouville type nonautonomous equations Preprint nlin.SI:1402.3692vl (2014).

[20] Adler V. E. and Startsev S. Ya. On discrete analogues of the Liouville equation Theoret. and Math. Phys. 121 (1999) 1484-1495.

[21] Zheltukhin K. and Zheltukhina N. On existance of an x-integral for a semi-discrete chain of hyperbolic type J. Phys.: Conf. Ser. 670 (2016) 434017. 